\section{Effektive Therapie bei Otitis externa}

— Die Entzündungen des Außenohrs tritt meist ohne vorangegangenen Infekt und in der Regel ohne Fieber auf. Das Allgemeinbefinden ist mit Ausnahme der als stark und stechend empfundenen Schmerzen, insbesondere bei mechanischer Erweiterung des Gehörgangs, nicht beeinträchtigt. Kennzeichnend sind Sekretbildung, starke Rötung und Schwellung des Gehörgangs. Nur bei vollständiger Verlegung kommt es zu einer Einschränkung der Schallleitungsfähigkeit.

Bei der Therapie der Otitis externa sollte eine rasche Intervention im Vordergrund stehen. Neben der Reinigung des Gehörgangs kommt dabei der Beseitigung der Erreger und abschwellenden Maßnahmen besondere Bedeutung zu. Die entsprechenden HNOLeitlinien empfehlen zur Bekämpfung der häufigsten Erreger Pseudomonas aeruginosa,

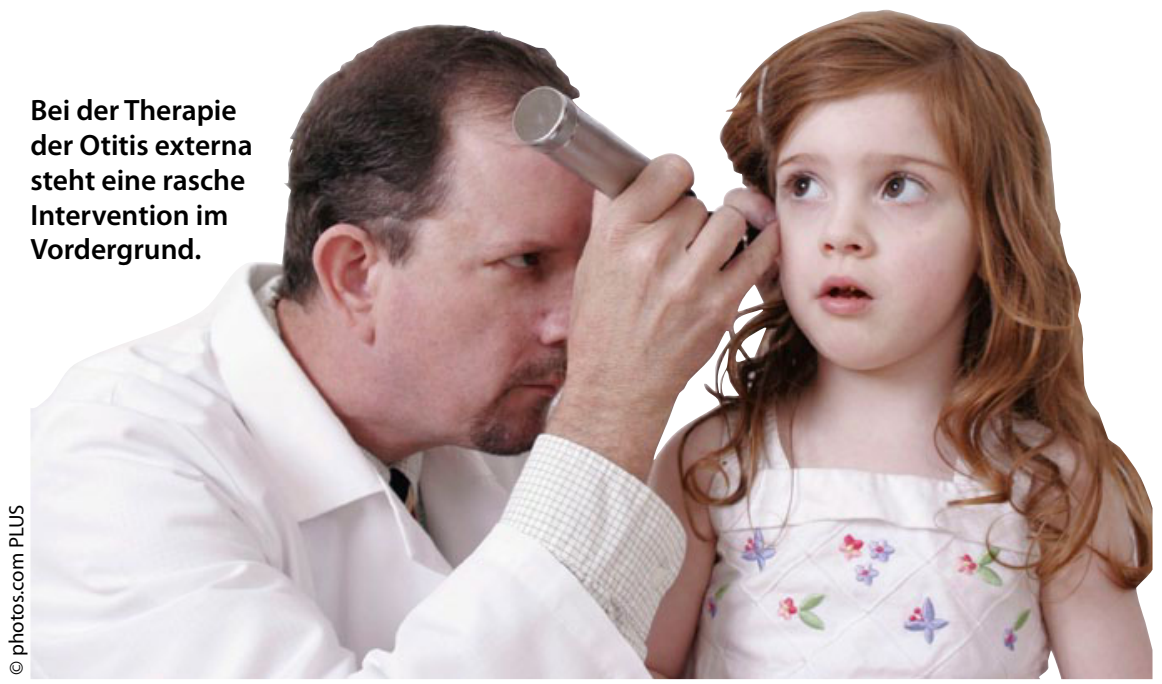

\title{
Immundefekte differenziert diagnostizieren
}

— Es gibt über 160 primäre angeborene Immundefekte, die sich je nach betroffener Komponente des Immunsystems in unterschiedliche Subgruppen unterteilen lassen. In Deutschland wird eine Prävalenz von $1: 2.000$ angenommen, wobei ca. $55 \%$ der Patienten einen Antikörpermangel aufweisen. Zu den typischen klinischen Anzeichen gehören: wiederkehrende bakterielle Infektionen mit häufig vorkommenden Erregern bzw. mit opportunistischen Erregern, unerklärbare Gedeihstörungen bei Kindern, Infektionen, die einer unverhältnismäßig langen Antibiotikatherapie bedürfen, sowie wiederkehrende Infektionen an mehr als einer Körperstelle.

Die frühzeitige Diagnose eines Immundefektes und der Zugang zu einer geeigneten Behandlung sind essenziell, um eine lange Erkrankung und Organschädigungen zu vermeiden. Die der Infektion zugrunde liegen-
Staphylococcus aureus und Proteus mirabilis eine antibiotische Lokaltherapie. Bewährt hat sich dabei der Wirkstoff Ciprofloxacin.

Mit Panotile ${ }^{\circledast}$ Cipro Ohrentropfen steht ein Präparat mit lipophilen und viskösen Eigenschaften (gute Haftung und Benetzung) und gut ans Ohrmilieu angepasstem pH 5,3-6,3 zur Verfügung. Es enthält zudem den Hilfsstoff Glycerol, der für seine abschwellende Wirkung bekannt ist. Als einzige Ciprofloxacin-haltige Ohrentropfen kommt Panotile ${ }^{\otimes}$ Cipro dank Einmaldosisbehältnissen ohne potenziell ototoxische Konservierungsstoffe aus.

Die hohe Therapiesicherheit spiegelt sich auch in der Tatsache wider, dass Panotile ${ }^{\circledR}$ Cipro als einziges Präparat auch zur leitliniengerechten lokalen Therapie der chronischeitrigen Otitis media mit Trommelfelldefekt zugelassen ist. Es kann auch bei liegenden Paukenröhrchen angewendet werden. Dem behandelnden Pädiater steht mit Panotile ${ }^{\circledR}$ Cipro also ein Arzneimittel zur Verfügung, das bei Otitis externa auch mit unklarem Trommelfellbefund sowie bei chronischer Otitis media mit Trommelfelldefekt oder liegenden Paukenröhrchen eine effektive und sichere Beseitigung der Erreger gewährleistet.

Nach Informationen von Pierre Fabre, Freiburg

eine medikamentöse Therapie (z. B. Chemotherapie) ausgelöst wurden, ist eine Infektionsprophylaxe durch Impfungen aufgrund des erhöhten Infektionsrisiko von hoher Bedeutung. Allerdings kann der Impferfolg durch die Immunsuppression vermindert sein, sodass auch hier Impferfolgskontrollen angezeigt sein können.

Binding Site ist ein auf Proteindiagnostik spezialisiertes Unternehmen, das sich auf die Erforschung, Entwicklung und Herstellung innovativer immundiagnostischer Tests für den Labormarkt spezialisiert hat. Mit seiner Kompetenz bei der Produktion hochspezifischer Antikörper sorgt Binding Site dafür, dass in klinischen Laboratorien und Forschungsstätten Reagenzien verfügbar sind, die eine deutliche Verbesserung bei der Diagnose und Behandlung von Patienten mit Störungen des Immunsystems ermöglichen.

Nach Informationen von Binding Site, Schwetzingen 